\title{
Respuesta fisiológica de plántulas de cacao (Theobroma cacao L.) sometidas a sombreamiento y a la aplicación de una enmienda orgánica
}

\author{
Physiological response to cocoa seedlings (Theobroma cacao L.) \\ shading and under an amendment application of organic
}

\section{Yuliana Banguero-García1, Glency Mosquera-Figueroa', Sindy Toro-Giraldo, Edna Leiva-Rojas ${ }^{2}$, Ramiro Ramírez-Pisco ${ }^{2}$}

\section{Resumen}

En la actualidad la alta demanda del cacao (Theobroma cacao L.) hace necesaria la ejecución de estudios que permitan conocer las condiciones de producción óptima de esta importante planta. Se evaluó la respuesta del cacao a diferentes intensidades lumínicas y nutricional, empleando tres niveles de luz: 100\%, $50 \%$ y $15 \%$ y aplicando tres dosis de la enmienda orgánica (Nutriplant): 1 ton/ha, 2 ton/ha y 4 ton/ha respectivamente. Los tratamientos se distribuyeron en un diseño experimental en bloques completamente al azar (DBC), con tres repeticiones. Se realizó un análisis de varianza y una prueba de comparación de medias de Duncan con un $\alpha=0.01$ por medio del software estadístico SAS. Las variables evaluadas fueron: longitud de raíz, área foliar, biomasa de raíz, hojas y tallo, conductancia estomática, transpiración, PAR y fotosíntesis neta. Se encontró que el cacao (Theobroma cacao L.) presenta una mejor respuesta sin estar sometido a una limitación de luz; esta afirmación se ve reflejada en que las plantas a plena exposición presentaron los valores más altos de biomasa de tallo, raíz y hojas, área foliar, fotosíntesis neta, transpiración y conductancia estomática; sin embargo, la aplicación de la enmienda orgánica (Nutriplant) no presentó ningún efecto en las variables nombradas. En las plantas expuestas a baja intensidad lumínica se observó una respuesta positiva en variables como altura y diámetro de copa ante la aplicación de la enmienda.

Palabras clave: Area foliar, Biomasa, Cacao (Theobroma cacao L.), Conductancia estomática, Enmienda orgánica, Fotosintesis neta, Luz, PAR, Presión parcial intercelular,

Transpiración, Temperatura de la hoja.
Ingeniera Agrónoma, Universidad Nacional de Colombia, sede Medellín. e-mail: lybanguerog@unal.edu.co gpmosqueraf@unal.edu.co sjtorog@unal.edu.co

2 Profesor Asociado, Universidad Nacional de Colombia, sede Medellín. e-mail: eileivar@unal.edu.co rramirez@unal.edu.co 


\section{Respuesta fisiológica de plántulas de cacao. Y Banguero-García et al.}

\section{Summary}

Currently the high demand for cocoa (Theobroma cacao L.) requires the implementation of studies to know the optimal production conditions of this important plant. We evaluated the response of cocoa to different light intensities and nutrition using three light levels: 100\%, $50 \%$ and $15 \%$ following three doses of organic amendment (Nutriplant): 1 ton/ha, 2 ton/ha and 4 ton/ha respectively. Treatments were distributed in an experimental design in randomized complete block (DBC) with three replications. An analysis of variance and means comparison test of Duncan with $\alpha=0.01$ using the SAS statistical software. The variables evaluated were: root length, leaf area, root biomass, leaf and stem, stomatal conductance, transpiration, PAR, and net photosynthesis. It was found that cocoa (Theobroma cacao L.) has a better answer without being subject to a limitation of light, this statement is reflected in the plants at full exposure showed the highest values of biomass of stem, root and leaves, leaf area, net photosynthesis, transpiration and stomatal conductance, but the application of organic amendment (Nutriplant) had no effect on the variables named. In plants exposed to low light intensity showed a positive response to variables such as height and crown diameter to the implementation of the amendment.

Keywords: Biomass, Cacao (Theobroma cacao L.), Intercellular partial pressure, Leaf temperature, Leaf area, Light, Organic amendment, Net photosynthesis, PAR, Stomatal conductance, Transpiration.

\section{Introducción}

Theobroma cacao es una planta leñosa de la familia Malvaceae (Alverson et al., 1999); es la única especie en el género Theobroma explotada por siglos (de Souza Claret et al., 1999). Crece en zonas tropicales húmedas y es considerado un cultivo importante a nivel mundial como materia prima para la fabricación del chocolate, el cual se produce a partir de las semillas (Despréaux, 2001). Está distribuida desde México hasta el Amazonas en una gran diversidad de ambientes que ha propiciado una alta cantidad de tipos o cultivares, producto de procesos de domesticación y adaptación a condiciones climáticas muy particulares. Esto ha producido una diversidad de cultivares con respuestas o tolerancia a variaciones y fluctuaciones de los parámetros microclimáticos (de Almeida y Valle, 2007).

El uso de enmiendas orgánicas se ha realizado como complemento al aporte de fuentes inorgánicas, como mejorador de las propiedades del suelo o en agricultura extensiva y orgánica. Sin embargo, el mal uso de estos residuos orgánicos, ya sea por altas dosis y/o inadecuada época de aplicación, ha sido asociado con la contaminación de aguas superficiales y subterráneas (Steenvoorden et al., 1986; Pain et al., 1986; Cameron et al., 1997), aire (Pain, 2000) y suelos (Wood et al., 1996; Mazzarino et al., 1997; Han et al., 2000; Gascho et al., 2001; Afyuni y Schulin, 2002; Pederson et al., 2002; Martínez et al., 2003; Sistani et al., 2004; Speir et al., 2004).

Por tanto, basados en la necesidad de conocer las condiciones de producción óptima de esta importante planta, analizando su comportamiento fisiológico y su desarrollo con base en dos aspectos muy discutidos e indispensables para su correcto manejo: la luz y la nutrición, se evaluó la respuesta del cacao (Theobroma cacao L.) a diferentes intensidades lumínicas y nutrición, empleando tres niveles de luz: 100\%, 50\% y 15\% y aplicando tres dosis de la enmienda orgánica (Nutriplant): 1 ton/ha, 2 ton/ha y 4 ton/ha respectivamente.

\section{Materiales y métodos}

Este proyecto se realizó en predios de la Universidad Nacional de Colombia, sede Medellín, 


\section{Bioetnia Volumen 12, 2015}

con una altitud de $1.467 \mathrm{msnm}$, temperatura promedio de $24^{\circ} \mathrm{C}$; la zona de vida pertenece a Bosque Húmedo Premontano, según la clasificación realizada por Holdridge en 1967 y con coordenadas geográficas de $6^{\circ} 15^{\prime} 46.67^{\prime \prime} \mathrm{N}$ y $75^{\circ} 34^{\prime}$ 42.06" O.

Se seleccionaron semillas de cacao a partir de la recolección de mazorcas maduras de la variedad IMC-67 proveniente de San Jerónimo, Antioquia; posteriormente las semillas germinaron en estopas de cabuya y aserrín alrededor de una semana, luego estas se establecieron a diferentes intensidades lumínicas: $100 \%, 50 \%$ y $15 \%$ y se sembraron en un sustrato al cual se le aplicó la enmienda orgánica (Nutriplant) en diferentes concentraciones: 1 ton/ ha, 2 ton/ha y 4 ton/ha respectivamente.

Se realizó un muestreo al mes y medio de sembradas las semillas. Las variables a considerar fueron: longitud de raíz, área foliar, biomasa de raíz, hojas y tallo, conductancia estomática, transpiración, PAR, fotosíntesis neta. Los tratamientos se distribuyeron en un diseño experimental en bloques completamente al azar (DBC), con tres repeticiones. Se realizó un análisis de varianza y una prueba de comparación de medias de Duncan con un $\alpha=0.01$, mediante el Software estadístico SAS.

\section{Resultados y discusión}

La altura de la planta no presentó diferencias significativas entre los tratamientos que se encontraban a plena exposición. Las plantas que se encontraban bajo sombra alcanzaron aproximadamente el doble de altura de las crecidas a mayor luminosidad, excepto el testigo que se encontraba a una intensidad lumínica del 15\% quien presentó una altura de $9.67 \mathrm{~cm}$, lo cual indica que al nutrir la planta se compensa la limitación lumínica. El incremento en altura que experimentan las plantas que se desarrollan bajo condiciones de sombreamiento es una respuesta morfogenética típica (Smith y Whitelam, 1990), correspondiente a un mayor alargamiento celular que ocurre como consecuencia de una escasa luminosidad, lo que constituye un mecanismo importante de adaptación (Rêgo y Possamai, 2006).

Las plantas a plena exposición y a una intensidad lumínica del 50\% no presentaron diferencias con respecto al diámetro de la copa; sin embargo, la plantas sometida a una intensidad lumínica del $15 \%$ mostraron un efecto positivo a la aplicación de la enmienda en todas las concentraciones, siendo esta mayor en 1 ton/ha.

Mayores longitudes de la raíz principal se obtuvieron en los tratamientos que se encontraban bajo sombra, la mayor longitud de esta fue para el testigo que se encontraba en un 50\% de luz; por lo tanto se puede decir que el Nutriplant no tiene efecto significativo en el desarrollo de las raíces en plántulas de cacao (Theobroma cacao L.). Mickovski y van Beek (2009) comentan que las plantas aumentan el tamaño de copa a lo largo del gradiente de precipitación, igualmente aumentan la profundidad de enraizamiento. Pocas plantas cultivadas son tan sensibles, como el cacao, a la deficiencia de agua y prácticamente todos sus procesos fisiológicos se afectan por la falta de humedad del suelo, se reduce la producción total y afecta la renovación de las hojas y flores (Alvim, 1977).

La aplicación de nutriplant ante una baja intensidad lumínica (15\%) y a plena exposición, no presentó un efecto significativo respecto a la biomasa de raíz, tallo y hojas. Sin embargo, con respecto a la biomasa de la raíz se pudo observar que ante la mayor luminosidad se obtuvieron mayores valores que bajo sombra; similarmente, en otras investigaciones se ha obtenido mayor masa radical en diversas especies leñosas expuestas a plena radiación solar (Robles Ortega et al., 2006). La alta biomasa radical propicia un mejor desempeño de las plantas una vez que se llevan a campo, sobre todo en áreas degradadas, pues la posibilidad de supervivencia sería superior en razón a la facilidad de sustentación y absorción 


\section{Respuesta fisiológica de plántulas de cacao. Y Banguero-García et al.}

de agua y nutrientes (de Almeida et al., 2005). El mayor contenido de biomasa total de la planta fue presentado en los tratamientos correspondientes al 100\% de luz. La mayor área foliar la presentó el testigo de esta condición lumínica, la disminución se pudo deber a la reducción en el número de hojas por planta (NeSmith y Duval, 1998).

La PAR obtuvo niveles más altos a plena exposición y no hubo respuesta de la planta a la aplicación de la enmienda orgánica (Nutriplant). Por otro lado el valor más alto de transpiración y de conductancia estomática se encontró en plena exposición (100\% luz), la primera pudo deberse porque la sombra reduce la tasa de transpiración de la hoja (Jaimez et al., 1999), mientras que la segunda pudo presentarse por temperaturas entre $20^{\circ} \mathrm{C}$ y $30^{\circ} \mathrm{C}$ en el suelo las cuales se consideran óptimas para mayores conductancias estomáticas (Amorin y Valle, 1993).

Experimentos conducidos en Brasil han demostrado que la fertilización del cacaotero bajo sombra solo produce pequeños incrementos en producción, mientras que la fertilización de cacao a plena exposición solar produce aumentos considerables en rendimiento de grano seco. La fotosíntesis es mucho más intensa en una plantación sin sombra y la respuesta a la fertilización es alta (Murray, 1982); experimentos conducidos en Ghana, demostraron que la aplicación de fertilizantes sin remoción de sombra incrementa la producción en

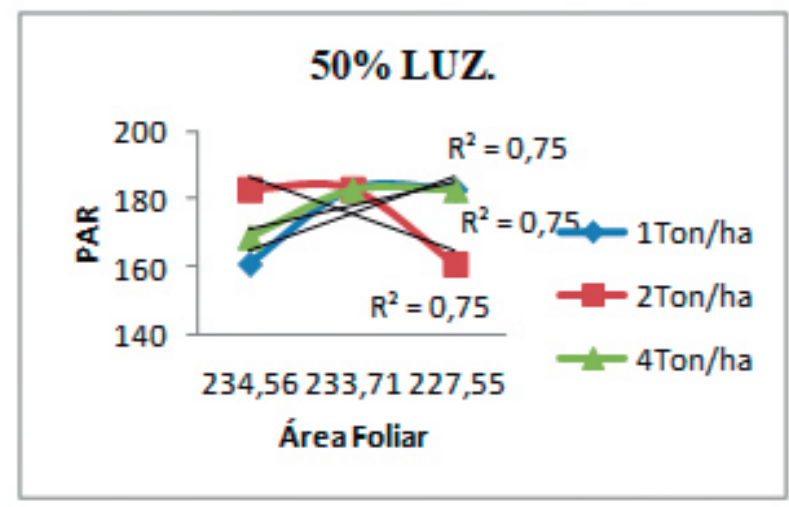

Figura 1. Relación entre PAR y área foliar a $\mathbf{5 0} \%$ luz. un 25\% (Pinto 1963, citado por Llano y Castaño 1977), corroborando los resultados obtenidos en la presente investigación (Tabla 1).

Hay una fuerte relación lineal entre las variables PAR y área foliar $\left(\mathrm{r}^{2}=0.75\right)$ a una intensidad lumínica de $15 \%$ y $50 \%$ en todas las concentraciones de la enmienda orgánica. Moraes-Neto et al. (2000) comentan que cuando una especie tiene la capacidad adaptativa para compensar la deficiencia de luz ocasionada por el sombreamiento, hay un incremento en el área foliar con lo cual se logra la máxima absorción de la luz incidente (Figuras 1 y 2).

La fotosíntesis neta y el área foliar a una intensidad lumínica del $100 \%$ y a concentraciones de la enmienda orgánica de 1 ton/ha y 4 ton/ha presentó valores de $\mathrm{r}^{2}$ mayores a $83 \%$, lo que indica una alta relación lineal entre las variables evaluadas; sin embargo, a una concentración de 2 ton/ha no hubo un coeficiente de determinación representativo, también evidenciado en una intensidad lumínica del 15\% y 50\%. De lo anterior se puede inferir que esta dosis de Nutriplant no presenta una influencia significativa entre el área foliar y la fotosíntesis neta (Figuras 3, 4 y 5).

Respecto a las Figuras 6, 7 y 8, no se puede deducir nada, porque su coeficiente de determinación es muy variable en cuanto a los tres niveles de intensidad lumínica y a las concentraciones de la enmienda orgánica (Nutriplant).

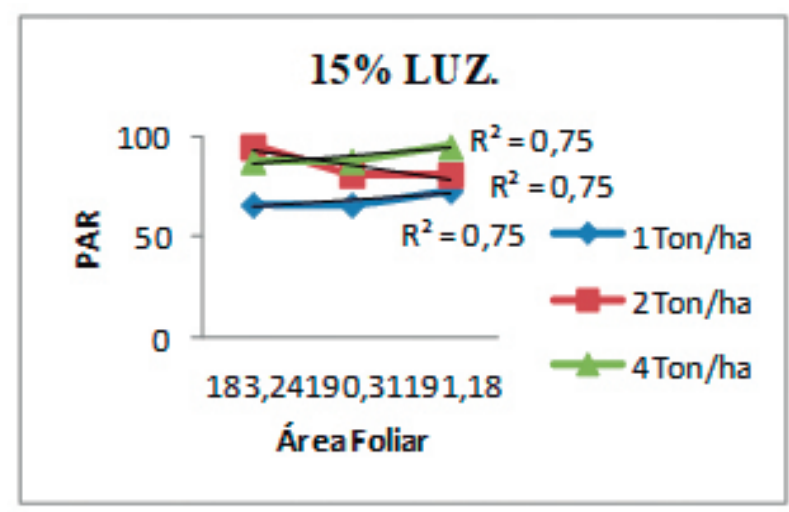

Figura 2. Relación entre PAR y área folia a $15 \%$ luz. 
Bioetnia Volumen 12, 2015

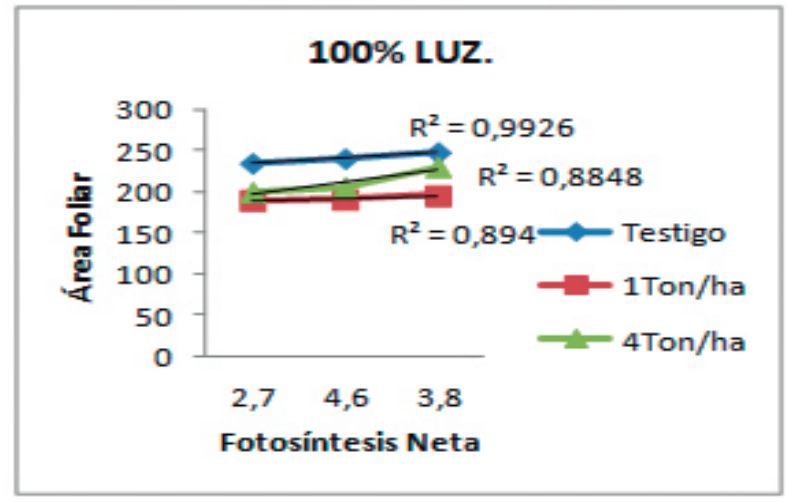

Figura 3. Relación entre área foliar y fotosíntesis neta a $100 \%$ luz.

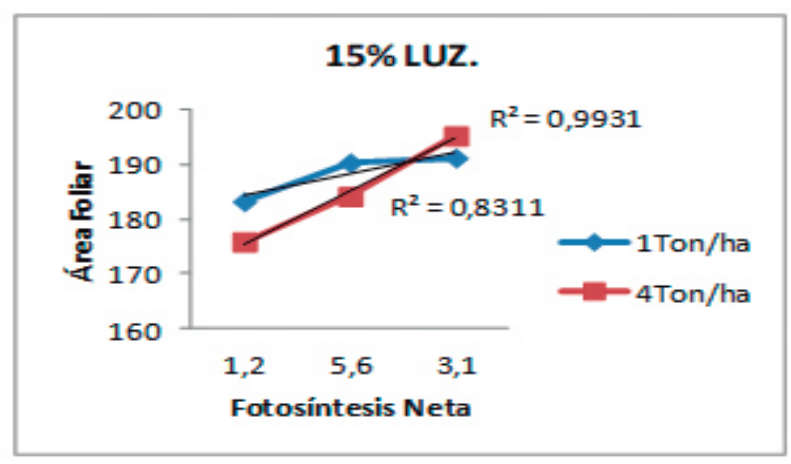

Figura 5. Relación entre área foliar y fotosíntesis neta a $15 \%$ luz.

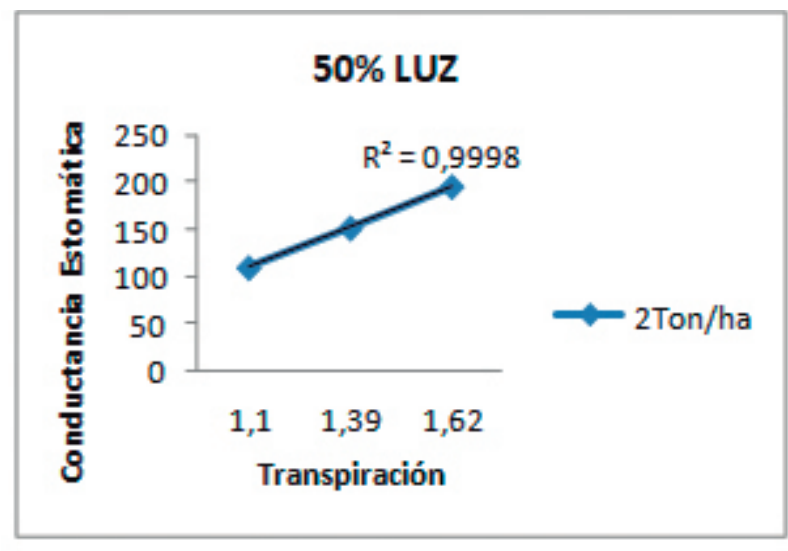

Figura 7. Relación entre conductancia estomática y transpiración a $50 \%$ luz.

\section{Conclusiones}

Se encontró que el cacao (Theobroma cacao L.) presenta una mejor respuesta sin estar sometido a una limitación de luz; esta afirmación se

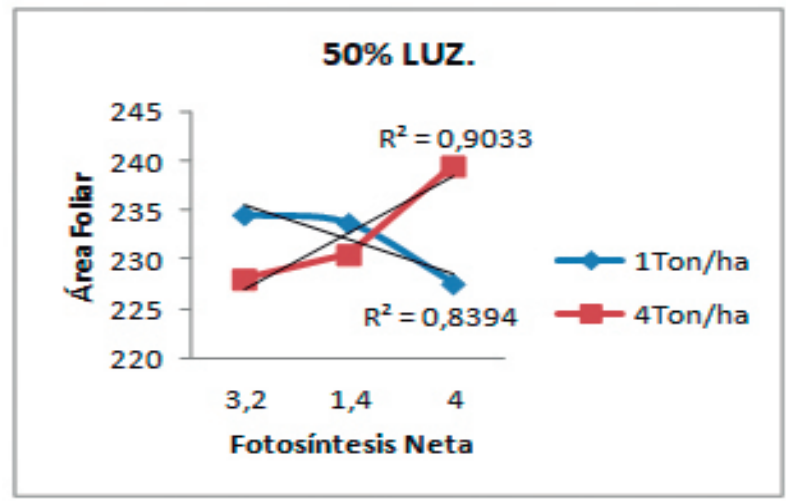

Figura4. Relación entre área foliar y fotosíntesis neta a $\mathbf{5 0 \%}$ luz.

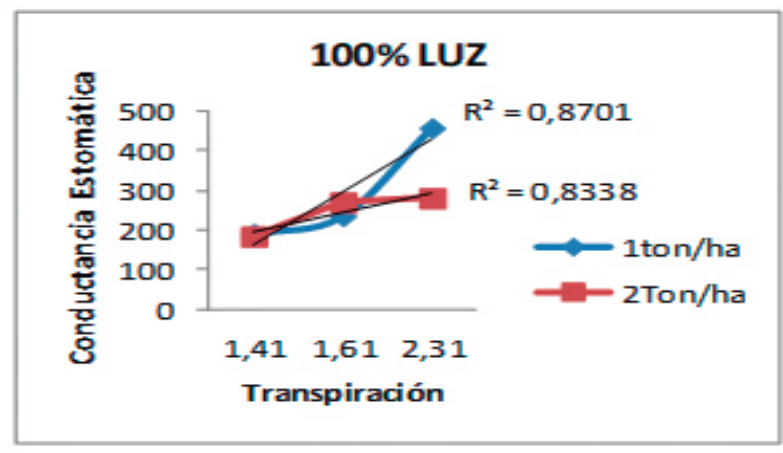

Figura 6. Relación entre conductancia estomática y transpiración a $100 \%$ luz.

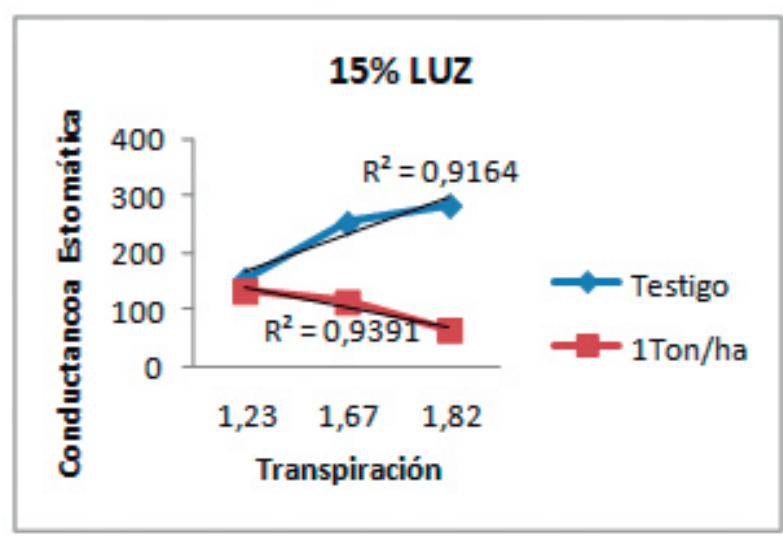

Figura 8. Relación entre conductancia estomática y transpiración a $15 \%$ luz.

ve reflejada en que las plantas a plena exposición presentaron los valores más altos de biomasa de tallo, raíz y hojas, área foliar, fotosíntesis neta, transpiración y conductancia estomática; sin embargo, la aplicación de la enmienda orgánica 


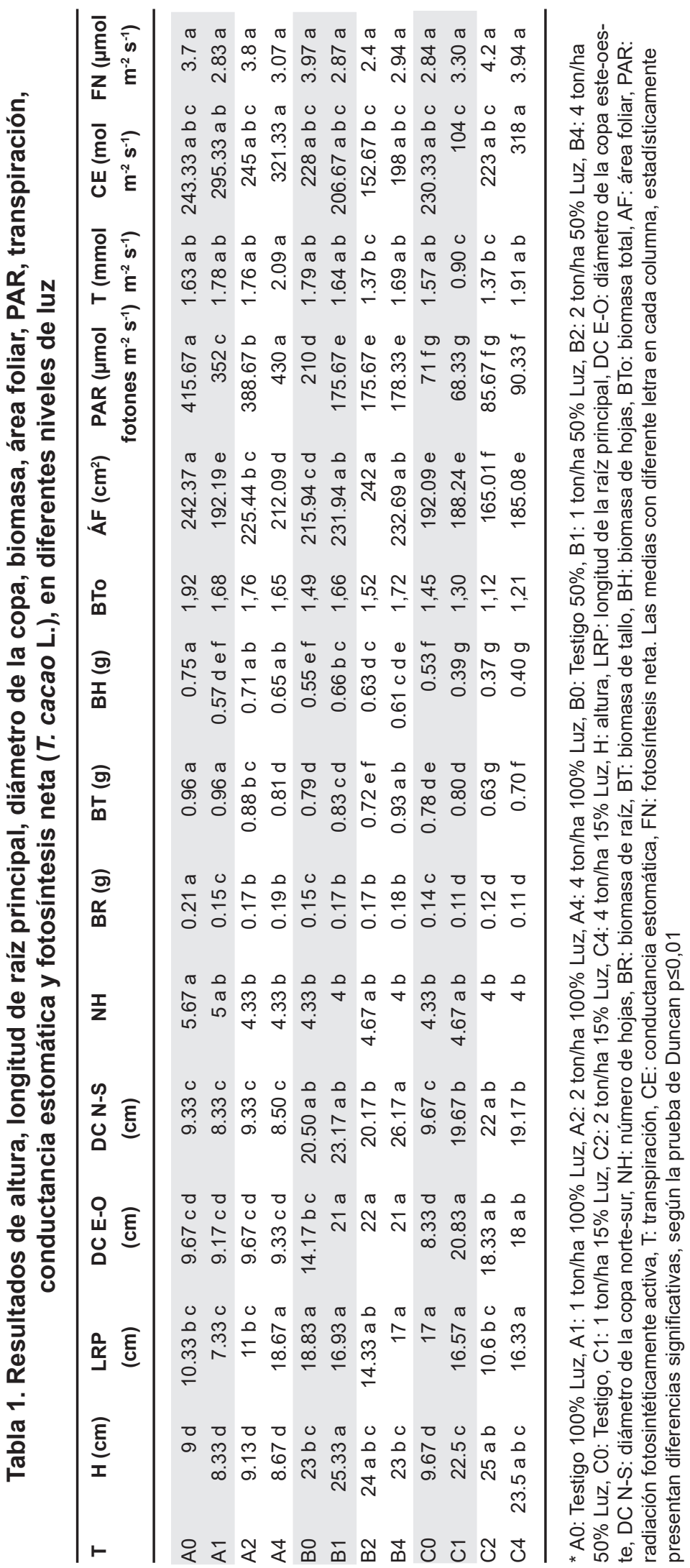

(Nutriplant) no presentó ningún efecto en las variables nombradas.

En las plantas expuestas a baja intensidad lumínica se observó una respuesta positiva en variables como altura y diámetro de copa ante la aplicación de la enmienda orgánica.

La aplicación de enmiendas nutricionales orgánicas a las plantas de cacao compensa la limitación lumínica a las cuales estas puedan estar sometidas, porque ejerce un efecto positivo sobre la variable altura.

Existe una alta relación lineal entre las variables evaluadas porque se determinó que la fotosíntesis neta y el área foliar a una intensidad lumínica del 100\% y a concentraciones de enmienda orgánica de 1 ton/ha y 4 ton/ha presentó valores de $r^{2}$ mayores a $83 \%$.

Las plantas de cacao expuestas a baja intensidad lumínica responden positivamente en variables como altura y diámetro de copa ante la aplicación de la enmienda orgánica.

\section{Glosario}

La PAR es la radiación fotosintéticamente activa(PAR) medida de la energía radiante; es importante para evaluar el efecto de la luz sobre el crecimiento vegetal. Esta designa la gama espectral (banda de onda) de la radiación solar de 400 a 700 nanómetros que los organismos fotosintéticos son capaces de utilizar en el proceso de fotosíntesis (McCree y Keith, 1972).

La fotosíntesis neta es la cantidad de $\mathrm{CO}_{2}$ por unidad de área foliar y de tiempo y se define como la cantidad neta de carbono que se incorpora al vegetal en un momento dado (Nouvellon et al., 


\section{Bioetnia Volumen 12, 2015}

2000; Bradford et al., 2005).

La conductancia estomática es la medida de la velocidad de paso de dióxido de carbono $\left(\mathrm{CO}_{2}\right)$ que entra o vapor de agua que sale a través de los estomas de una hoja. La tasa de conductancia estomática, o su resistencia inversa estomática, está directamente relacionada con la resistencia de la capa límite de la hoja y el gradiente de concentración absoluta de vapor de agua de la hoja a la atmósfera (Taiz y Zeiger, 1991).

La biomasa es la producción de biomasa total, es una función de la radiación solar fotosintéticamente activa incidente (RFAinc) de la eficiencia con la que el cultivo la intercepta y con que la planta utiliza la energía interceptada (EUR) para convertirla en materia seca. Tanto la eficiencia de intercepción como la RFA interceptada (RFAint) tienen una relación directa con el índice de área foliar (Heichel et al., 1988).

\section{Literatura citada}

Afyuni M, Schulin R. 2002. Repetitive and residual effects of sewage sludge application on extractability and plant uptake of $\mathrm{Cu}, \mathrm{Zn}, \mathrm{Pb}$ and $\mathrm{Cd}$. pp. 250-5. Proceedings of $12^{\text {th }}$ International Soil Conservation Organization Conference, Beijing, China. URL disponible en: $\underline{\text { http:// }}$ tucson.ars.ag.gov/isco/isco12/VolumeIII/RepetitiveandResidualEffects.pdf

deAlmeida LS de, Maia N da, Ortega AR, Angelo AC. 2005. Crescimento de mudas de Jacaranda puberula Cham. en viveiro submetidas a diferentes niveis de luminosidade. Ciencia Florestal 15 (3): 323-9. URL disponible en: http://www.scielo.br/pdf/cflo/v15n3/1980-5098cflo-15-03-00323.pdf

de Almeida AA, Valle R. 2007. Ecophysiology of the cacaa tree. Braz J Plant Physiol. 19 (4): 425-48. URL disponible en: http://www.scielo.br/scielo.php?scrip$\mathrm{t}=$ sci arttext\&pid=S1677-04202007000400011

Alverson WS, Whitlock BA, Nyffeler R, Bayer C, Baum DA. 1999. Phylogeny of the core Malvales: evidence from ndhF sequence data. Am J Bot. 86 (10):1474-86. https://www.ncbi.nlm.nih.gov/pubmed/10523287

Alvim PT. 1977. Cacao. pp. 279-313. In: Alvim PT, Kozlowski TT (eds.). Ecophysiology of tropical crops. New York:Academic Press. URL disponible en: https://www. elsevier.com/books/ecophysiology-of-tropical-crops/ alvim/978-0-12-055650-2

Amorin SMC, de Valle RR. 1993. Absorçao e resistencia ao movimento da agua no cacaueiro. Pesquisa Agropecuaria Brasilera. 28 (8): 907-13. URL disponible en: https://www.alice.cnptia.embrapa.br/handle/doc/105452

Bradford JB, Hickec JA, Lauenrothb WK. 2005. The relative importance of light-use efficiency modifications from environmental conditions and cultivation for estimation of large-scale net primary productivity. Remote Sensing of Environment. 96 (2): 246-55. URL disponible en: http://www.sciencedirect.com/science/article/pii/ S003442570500088X

Cameron KC, Di HJ, McLaren RG. 1997. Is soil appropriate dumping ground for our wastes? Aust J Soil Res. 35 (5): 995-1035.

de Souza ClaretAdasG, da Silva SE, Tavares AM, Rodrigues AM, Rodrigues MdoR. 1999. A cultura do cupuaçu [Theobroma grandiflorum (Will ex Spreng.) Schum.]. In:Embrapa Amazônia Ocidental. Circular Técnica $N^{\circ}$ 2. URL disponible en: https://www.infoteca.cnptia.embrapa.br/infoteca/bitstream/doc/670563/1/circtec2.pdf

Despréaux D. 2001. Overview on perennial cultures. Plantation Research and Development; pp.95.

Gascho GJ, Hubbard RK, Brenneman TB, Johnson AW, Sumner DR, Harris GH. 2001. Effects of broiler litter in an irrigated, double-cropped, conservation-tilled rotation. Agron J. 93 (6): 1315-20. URL disponible en: https://dl.sciencesocieties.org/publications/aj/ abstracts $/ 93 / 6 / 1315$ ? access $=0 \& v i e w=p d f$

Han FX, Kingery WL, Selim HM, Gerard PD. 2000. Accumulation of heavy metals in a long-term poultry waste-amended soil. Soil Sci. 165 (3): 260-8. URL disponible en: http://journals.lww.com/soilsci/ Abstract/2000/03000/ACCUMULATION_OF HEAVY_METALS_IN_A_LONG_TERM.8.aspx?trendmd-shared $=0$

Heichel GH, Delaney RH, Cralle HT. 1988. Carbono assimilation, partitioning and utilization. pp. 196-227. In: Hanson AA, Barnes DK, Hill RR (eds.) Alfalfa and Alfalfa Improvement-Agronomy Monograph $N^{\circ}$ 29. ASA-CSSA-SSSA. URL disponible en: https:// dl.sciencesocieties.org/publications/books/abstracts/ agronomymonogra/alfalfaandalfal $/ 195$ ? access $=0 \&-$ view $=$ pdf

Holdridge, L. R. 1967. Life zone ecology. San José: Tropical Science Center; 1982. URL disponible en: http://reddcr.go.cr/sites/default/files/centro-de-documentacion/ holdridge_1966 _ life_zone_ecology.pdf

Jaimez RE, Rada F, García-Nuñez C. 1999. The effects of irrigation frequency on water and carbon relations in three cultivars of sweet pepper (Capsicum chinense Jacq), in tropical semiarid region. Sci Hortic. 81: 301-8. 


\section{Respuesta fisiológica de plántulas de cacao. Y Banguero-García et al.}

Llano AO, Castaño MC. 1977. Segunda etapa del ensayo sobrefertilización en cacao (Theobroma cacao L.) en la hacienda Calamar. Tesis de grado para optar el título de Ingeniero Agrónomo. Manizales: Universidad Caldas.

McCree KJ. 1972. Test of current definitions of photosynthetically active radiation against leaf photosynthesis data. Agricultural and Forest Meteorology. 10: 443-53. URL disponible en: http://www.sciencedirect.com/ science/article/pii/0002157172900453

Martínez F, Cuevas G, Calvo R, Walter I. 2003. Biowaste effects on soil and native plants in a semiarid ecosystem. J Environ Qual. 32 (2): 472-9. URL disponible en: https://www.ncbi.nlm.nih.gov/pubmed/12708670

Mazzarino MJ, Walter I, Costa G, Laos F, Roselli L, Satti P. 1997. Plant response to fish farming wastes in volcanic soils. J Environ Qual. 26: 522-8.

Mickovski SB, van Beek LPH. 2009. Root morphology and effects on soil reinforcement and slope stability of young vetiver (Vetiveria zizanioides) plants grown in semi-arid climate. Plant Soil. 324: 43-56. URL disponible en: http://www.academia.edu/4784119/ Root morphology and effects on soil reinforcement and slope stability of young vetiver Vetiveria_zizanioides_plants_grown_in_semi-arid_climate

Moraes-Neto S, Gonçalves J, Takaki M, Cenci S. 2000. Crescimento de mudas de algumas espécies arbóreas que ocorrem na Mata Atlántica, em fução do nível de luminosidade. Revista Avore 24 (1): 35-45.

Murray DB. 1982. Sombra y nutrición. pp. 13-159. En: Cacao. $3^{\mathrm{a}}$ ed. Ciudad de México: Compañía Editorial Continental (CECSA).

NeSmith DS, Duval JR. 1998. The effect of container size. HortTechnology 8 (4): 1-4. URL disponible en: http://swfrec.ifas.ufl.edu/docs/pdf/veg-hort/transplant/ trans_cs1.pdf

Nouvellon Y, Lo Seen D, Rambal S, Bégué A, Moran MS, Kerr Y, Qi J. 2000. Time course of radiation use efficiency in a shortgrass ecosystem: consequences for remotely sensed estimation of primary production. Remote Sensing of Environment. 71 (1): 43-55. URL disponible en: http://www.sciencedirect.com/science/ article/pii/S0034425799000632

Robles Ortega A, de Almeida LS, da Maia N, Camargo A. 2006. Avalição do crecimento de mudas Psidium cattleianum Sabine a diferentes níveis de sombreamento em viveiro. Cerne. 12 (3): 300-8. URL disponible en: http://www.redalyc.org/pdf/744/74412311.pdf

Pain BF, Smith KA, Dyer CJ. 1986. Factors affecting the response of cut grass to the nitrogen content of dairy cow slurry. Agricultural Wastes 17 (3): 189-202. URL disponible en: http://www.sciencedirect.com/science/ article/pii/0141460786900934

Pain BF. 2000. Control and utilization of livestock manures. pp. 343-364. In: Hopkins A. (ed.). Grass: its production and utilization. $3^{\text {rd }}$ ed. Oxford: British Grassland Society; 456 pp.

Pederson GA, Brink GE, Fairbrother TE. 2002. Nutrient uptake in plant parts of sixteen forages fertilized with poultry litter: nitrogen, phosphorus, potassium, copper, and zinc. Agronomy J. 94 (4): 895-904.

Rêgo GM, Possamai E. 2006. Efeito do sombreamento sobre o teor de clorofila e crescimento inicial do jequitibá-rosa. Bol Pesq. 53: 179-94. URL disponible en: https://ainfo.cnptia.embrapa.br/digital/bitstream/ CNPF/42125/1/BPF 53 p179-194.pdf

SAS Institute Inc. SAS 9.1.3. Help and documentation. Cary: SAS Institute Inc., 2000-2004.

Sistani KR, Brink GE, Adeli A, Tewolde H, Rowe DE. 2004. Year-round soil nutrient dynamic from broiler Litter application to bermudagrass. Agron J. 96: 52530. URL disponible en: https://www.ars.usda.gov/ research/publications/publication/?seqNo115=148053

Smith H, Whitelam G. 1990. Phytocrome, a family of photoreceptors with multiple physiological roles. Plant Cell Environ. 13 (7): 695-707. URL disponible en: $\underline{\text { http://on- }}$ linelibrary.wiley.com/doi/10.1111/j.1365-3040.1990. tb01084.x/epdf?r3 referer=wol\&tracking action $=$ preview click\&show checkout $=1 \&$ purchase refer$\underline{\text { rer }=\text { www.google.com.co\&purchase site license }=\text { LI- }}$ CENSE DENIED

Speir TW, Horswell J, van Schaik AP, McLaren, RG Fietje G. 2004. Composted biosolids enhance fertility of a sandy loam soil under dairy pasture. Biol Fertil Soils. 40 (5): 349-58. URL disponible en: https://link.springer. com/article/10.1007\%2Fs00374-004-0787-6?LI=true

Steenvoorden J, Fonck H, Oosterom HP. 1986. Losses of nitrogen from intensive grassland systems by leaching and surface runoff. pp. 85-97. In: Van Der Meer HG, Ryden JC, Ennik GC (eds.). Nitrogen fluxes in intensive grassland systems. Developments in Plant and Soil Sciences. 23. Dordrecht: Springer. URL disponible en: https://link.springer.com/chapter/10.1007 \%2F978-94-009-4394-0_8

TaizL,ZeigerE. 1991. Plantphysiology. pp. 92-5. Redwood City: The Benjamin/Cummings Publishing Company, Inc; 559 pp.

Wood BH, Wood W, Yoo KH, Yoon KS, Delaney DP. 1996. Nutrient accumulation and nitrate leaching under broiler litter amended corn fields. Communications in Soil Science and Plant Analysis. 27 (15-17): 2875-94. 